\title{
X-RAY AND RADIO CONSTRAINTS ON THE MASS OF THE BLACK HOLE IN SWIFT J164449.3+573451
}

\author{
J. M. Mïller ANd K. GÜLTEKIN \\ Department of Astronomy, University of Michigan, 500 Church Street, Ann Arbor, MI 48109, USA; jonmm@umich.edu \\ Received 2011 June 13; accepted 2011 July 27; published 2011 August 12
}

\begin{abstract}
Swift J164449.3+573451 is an exciting transient event, likely powered by the tidal disruption of a star by a massive black hole. The distance to the source, its transient nature, and high internal column density serve to complicate several means of estimating the mass of the black hole. Utilizing newly refined relationships between black hole mass, radio luminosity, and X-ray luminosity, and de-beaming the source flux, a weak constraint on the black hole mass is obtained: $\log \left(M_{\mathrm{BH}} / M_{\odot}\right)=5.5 \pm 1.1(1 \sigma$ confidence). The confidence interval is determined from the current intrinsic scatter in the relation, which includes effects from X-ray variability and accretion modes. This mass range is broad, but it includes low values that are consistent with some variability arguments, and it safely excludes high-mass values where it becomes impossible for black holes to disrupt stars. Future refinements in relationships between black hole mass, radio luminosity, and X-ray luminosity will be able to reduce the uncertainty in related mass estimates by a factor of two, making this technique comparable to estimates based on the $M-\sigma$ relationship. Possible difficulties in placing such events on the fundamental plane, a potential future test of their suitability, and uncertainties in mass stemming from variable X-ray emission are discussed. As near- and longer-term survey efforts such as Pan-STARRS, LSST, LOFAR, the Square Kilometer Array, and eROSITA begin to detect many tidal disruption events, black hole mass estimates from combined X-ray and radio observations may prove to be very pragmatic.
\end{abstract}

Key words: accretion, accretion disks - black hole physics

Online-only material: color figure

\section{INTRODUCTION}

Swift J164449.3+573451 was originally detected on 2011 March 28 as a gamma-ray burst, GRB 110328 A, via the Swift $X$-ray Observatory. The properties of the source, including its variability and longevity, quickly made it clear that the source must be a new kind of transient event. Optical observations soon revealed a redshift of $z=0.354$ for the host galaxy (Levan et al. 2011a) and a position close to the galactic center. Based on the source properties, including variations in the X-ray spectrum and flux, it was suggested that the transient source may be powered by the tidal disruption of a star by a supermassive black hole (SMBH; Bloom et al. 2011a), viewed close to the axis of a jet. Subsequent and detailed studies support this interpretation (e.g., Bloom et al. 2011b; Burrows et al. 2011; Levan et al. 2011b; Krolik \& Piran 2011).

The mass of the black hole that likely disrupted the star is an interesting and important question. Rees (1988) predicts that the disruption of normal stars is possible for black holes for masses below $10^{8} M_{\odot}$ and describes observational hallmarks of the aftermath. Changes in the gravitational force across the diameter of a star are too gentle for black holes above this mass range, and stars are then swallowed whole.

In the case of Swift J164449.3+573451, its distance makes it impossible to trace stellar orbits close to the compact object and to establish a mass by resolving the dynamical sphere of influence (see, e.g., Gültekin et al. 2009b). A mass estimate via the $M-\sigma$ relationship is possible but may not be easy given the source distance, flux, and unknown host morphology. Other methods for estimating black hole masses are tied to direct primary masses and the $M-\sigma$ relationship, such as reverberation mapping (e.g., Peterson et al. 2004). However, this may also be complicated by strong absorption along the line of sight and by the transient nature of the source.
A new method for estimating black hole masses relies on the "fundamental plane" of black hole accretion (e.g., Merloni et al. 2003; Falcke et al. 2004). The plane reveals a relationship between radio luminosity, X-ray luminosity, and black hole mass. Radio luminosity serves as a proxy for jet power and Xray luminosity for accretion power. The ability of the plane to predict a black hole mass based on radio and X-ray luminosity estimates was sharpened by Gültekin et al. (2009a), wherein only black holes with direct primary masses are used. Inverting the plane to give masses shows great promise: current mass estimates are only a factor of two less certain than estimates from the $M-\sigma$ relationship itself, and the addition of more sources and simultaneous radio and X-ray observations may make it a comparably reliable predictor. The plane was recently used to estimate the mass of the central black hole in the dwarf starburst galaxy Henize 2-10 (Reines et al. 2011).

It has been unclear how to treat beamed sources, with respect to the fundamental plane. The power of the plane is that it gives information on the central engine-how accretion inflow and relativistic jet outflows are coupled. Jetted sources, in contrast, may not allow a clean view of the central engine. Plotkin et al. (2011) have recently shown that beamed sources-including BL Lac objects-fall on the fundamental plane once their fluxes are de-beamed. This methodology may not be uniformly applicable to other sources, but it demonstrates that beamed sources follow the same relation as others once a careful analysis of the relativistic effects is made. Coupled with the ability of the refined plane to give mass estimates, such treatments enable an estimate of the black hole mass in sources such as Swift J164449.3+573451.

In the sections that follow, we detail a mass estimate for Swift J164449.3+573451 based on simultaneous radio and $\mathrm{X}$-ray observations and fundamental plane relations. This estimate requires several assumptions; possible drawbacks and a 
possible test of whether or not such sources can be placed on the fundamental plane are included in Section 4.

\section{DATA SELECTION AND REDUCTION}

To place Swift J16449.3+573451 on the fundamental plane of black hole accretion, radio and X-ray luminosity points are required. The importance of obtaining contemporaneous radio and X-ray points in drawing physical inferences from the plane, and in reducing its internal scatter, is becoming clear (e.g., King et al. 2011; Jones et al. 2011). We therefore selected publicly available fluxes separated by the smallest possible margin.

Bower et al. (2011) observed Swift J16449.3+573451 using the Very Long Baseline Array on 2011 April 1, starting at 05:30 UT. They measure a flux density of $1.7 \pm 0.1 \mathrm{mJy}$ at $8.4 \mathrm{GHz}$, with an upper limit of just $17 \%$ on variations in the flux density within the $4 \mathrm{hr}$ observation. In a more recent analysis, Zauderer et al. (2011) report a flux density of $0.82(2) \mathrm{mJy}$ at $4.9 \mathrm{GHz}$ on April 1.27 (6:48 UT). The fundamental plane is constructed using flux density measurements in different bands, shifted to $5 \mathrm{GHz}$. Also on 2011 April 1, the Swift/ X-Ray Telescope (XRT) observed the source in PCW3 mode for a total of $21 \mathrm{ks}$, starting at 00:52 UT. Thus, both the $8.4 \mathrm{GHz}$ and $4.9 \mathrm{GHz}$ radio measurements had brief periods of overlap with this early X-ray observation.

The XRT data were reduced using the latest HEASOFT suite (version 6.10) and a fully up-to-date CALDB. Source events were extracted from the cleaned PCW3 event list in an annular region centered on the source, with an inner radius of 10 pixels and an outer radius of 40 pixels. This region was chosen to prevent photon pile-up distortions to the spectrum and flux. Background events were extracted from a circular region with a radius of 20 pixels, located well away from the source. The tool "xselect" was used to create source and background spectra. The standard redistribution matrix file from the calibration database was used, and an ancillary response function was generated using the tool "xrtmkarf."

The spectra were grouped to require at least 10 counts per spectral bin using the tool "grppha," and the spectrum was fit using XSPEC version 12 (Arnaud \& Dorman 2000). The spectrum below $0.5 \mathrm{keV}$ and above $10.0 \mathrm{keV}$ (in the observed frame) was ignored owing to calibration uncertainties in those bands. Neutral absorption along the line of sight was fit using the "ztbabs" model (Wilms et al. 2000), which places photoelectric absorption edges at the energy appropriate given the redshift of the source. This simple model gives an adequate fit, $\chi^{2} / v=620.8 / 529=1.17$ (see Figure 1). A high column density is measured, $N_{\mathrm{H}}=1.32(4) \times 10^{22} \mathrm{~cm}^{-2}$. The powerlaw photon index is measured to be $\Gamma=1.73(3)$; this slope is canonical for Seyfert-1 active galactic nucleus (AGN; see, e.g., Nandra et al. 1997). A power-law flux normalization of $9.1(3) \times$ $10^{-3} \mathrm{ph} \mathrm{cm}^{-2} \mathrm{~s}^{-1}$ is obtained. This gives a $2-10 \mathrm{keV}$ (observed frame) flux of $3.4(1) \times 10^{-11} \mathrm{erg} \mathrm{cm}^{-2} \mathrm{~s}^{-1}$, corresponding to a 2-10 keV flux of 2.9(1) $\times 10^{-11} \mathrm{erg} \mathrm{cm}^{-2} \mathrm{~s}^{-1}$ in the emitted frame. All of the above errors correspond to $1 \sigma$ uncertainties.

\section{MASS ESTIMATION}

Although the flux density measurement at $8.4 \mathrm{GHz}$ (Bower et al. 2011) has move overlap with the X-ray observation employed in this work, the flux density of $0.82(2) \mathrm{mJy}$ at 4.9 GHz reported by Zauderer et al. (2011) is more relevant. The fundamental plane (FP) of Merloni et al. (2003), for instance, was constructed by gathering radio core measurements

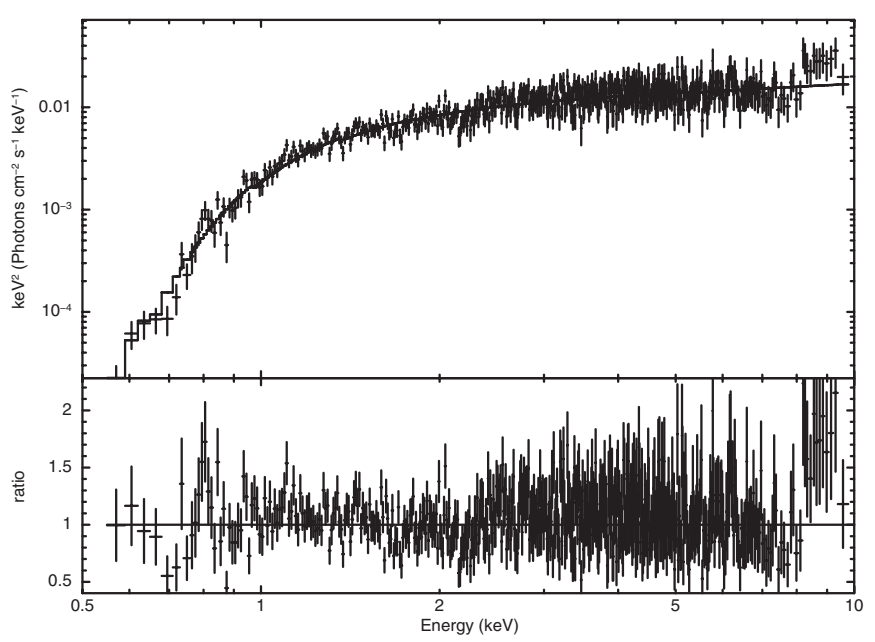

Figure 1. Unfolded XRT spectrum of Swift J164449.3+573451, obtained on 2011 April 1. The top panel shows the unfolded spectrum and the bottom panel shows the ratio of the data to a simple absorbed power-law model with $\Gamma=1.73(3)$. The turnover at low energy is the result of a high column density, $N_{\mathrm{H}}=1.32(4) \times 10^{22} \mathrm{~cm}^{-2}$. This basic spectral model allows for a good characterization of the total X-ray flux in the XRT band.

at different frequencies and shifting them to $5 \mathrm{GHz}$. The FP contains a broad range of sources, from LINERs with core emission that can be optically thick to Seyferts with emission that is optically thin (for a review of AGN core properties, see, e.g., Ho 2008).

The sources in FP relations are typically closer than Swift $\mathrm{J} 164449.3+573451$, and in order to use the FP, then, we need $L_{R}=v L_{v}$ at $5 \mathrm{GHz}$ in the observed frame. Zauderer et al. (2011) report on a number of radio observations on 2011 March 30 and find $F_{v} \propto v^{1.3 \pm 0.1}$. Based on their tables, we confirmed that the same index holds on 2011 April 1, and shifted the flux density to $5 \mathrm{GHz}$ in the observed frame for this assumed spectrum. At a redshift of $z=0.3534$ (Levan et al. 2011b) the luminosity distance is $D_{L}=1.8 \mathrm{Gpc}$ for an assumed cosmology of $h=0.71, \Omega_{m}=0.27$, and $\Omega_{\Lambda}=0.73$. Thus assuming isotropic emission, $L_{R}=8.26 \times 10^{39} \mathrm{erg} \mathrm{s}^{-1}$. For the same distance, the rest frame $2-10 \mathrm{keV}$ flux corresponds to an isotropic luminosity of $L_{X}=1.12 \times 10^{46} \mathrm{erg} \mathrm{s}^{-1}$.

In the case of Swift J164449.3+573451, observations of radio scintillation by Zauderer et al. (2011) are helpful: a Lorentz factor of $\Gamma \simeq 5$ is implied by their measurements. We have assumed this value of $\Gamma$ in de-boosting the emission observed from Swift J164449.3+573451. Special care is needed: the specific de-boosting calculations employed by Plotkin et al. (2011) assume optically thin emission (also see Lind \& Blandford 1985) and may not appropriate for Swift J164449.3+573451, or at least not at early times. An expression for beaming due to relativistic motions for a generic spectral index is given by $F=F_{0}(1-\beta \mu)^{-(2+\alpha)}$ (where $F$ is the flux observed, $F_{0}$ is the flux emitted in the source frame, $\beta=v / c$ and derives from $\Gamma, \mu=\cos (\theta)$, and $\alpha$ is the spectral index of the emitter; see, e.g., Peacock 1999). Assuming that $\mu=1$, we derive a correction factor of 15.5 . The intrinsic luminosities are then $L_{R}=5.3 \times 10^{38} \mathrm{erg} \mathrm{s}^{-1}$ and $L_{X}=7.2 \times 10^{44} \mathrm{erg} \mathrm{s}^{-1}$.

Although many FP fits exist for different source collections and aims (e.g., Merloni et al. 2003; Falcke et al. 2004; Yuan \& Cui 2005; Gültekin et al. 2009a; Plotkin et al. 2011), we use the FP from Gültekin et al. (2009a), which was fit using only sources with dynamically determined black hole masses. Gültekin et al. (2009a) gave several fits using different samples and statistical 


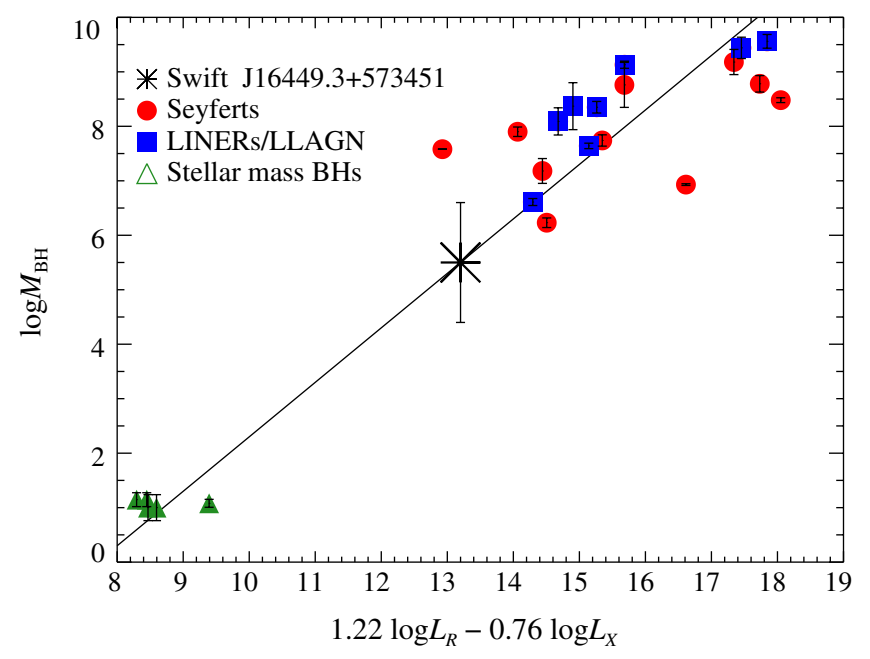

Figure 2. Inverted fundamental plane of black hole accretion. The sources with measured masses are from Gültekin et al. (2009a). The position of Swift $\mathrm{J} 164449.3+573451$ is shown with a cross. With the assumption that the FP holds for this new and interesting source, the observed X-ray and radio luminosities imply that the black hole has mass $\log M=5.5 \pm 1.1$.

(A color version of this figure is available in the online journal.)

methods. We use the fit defined by their Equations (1) and (10), which has the same functional form as fits by Merloni et al. (2003) and includes stellar-mass black holes. We chose this version because it should interpolate between the $10^{7}$ and $10^{1} M_{\odot}$ black holes. The mass predictor fit is only valid in its fitting domain of $M>10^{6} M_{\odot}$ and $L_{X}<10^{43} \mathrm{erg} \mathrm{s}^{-1}$. The fit we are using can be written as

$$
\begin{aligned}
\log L_{R}= & (6.31 \pm 0.21)+(0.82 \pm 0.08) \log M \\
& +(0.62 \pm 0.10) \log L_{X},
\end{aligned}
$$

where $L_{R}$ and $L_{X}$ are in units of erg s ${ }^{-1}$ and $M$ is in solar mass units. The rms intrinsic scatter of this relation is $0.88 \mathrm{dex}$ in the $\log L_{R}$ direction. This may be inverted to write

$$
\log M=1.22\left(\log L_{R} \pm 0.88\right)-0.76 \log L_{X}-7.70,
$$

dropping coefficient uncertainties since the scatter dominates. Using this relation with the luminosities above, we find $\log M=$ $5.5 \pm 1.1$, corresponding to $M=3.2 \times 10^{5} M_{\odot}$ with a $68 \%$ confidence range of $(0.25-40) \times 10^{5} M_{\odot}$. In Figure 2, we show Swift J164449.3+573451 on the FP with SMBHs and stellarmass black holes.

We may compare this mass estimate to a prediction based on the relation between black hole mass and the host galaxy's bulge luminosity, the $M-L$ relationship. In the $V$ band, it is given by $\log M=(8.95 \pm 0.11)+(1.11 \pm 0.18) \log \left(L_{V} / 10^{11}\right)$, where $L_{V}$ is given in units of $V$-band solar luminosities and the rms intrinsic scatter is $0.38 \pm 0.09$ dex (Gültekin et al. 2009b). The host galaxy of Swift J164449.3+573451 has $L_{B}=10^{9.20}$ (Leloudas et al. 2011; Burrows et al. 2011), which is very close to rest frame $V$ band. Thus, the mass predicted by the $M-L$ relation is $\log M=6.95 \pm 0.38$, corresponding to $M=9 \times 10^{6} M_{\odot}$ with a $68 \%$ confidence interval of $(3.7-21) \times 10^{6} M_{\odot}$. The $H$ band luminosity of $L_{H}=10^{9.58}$ is very close to rest frame $K$ band and predicts (Marconi \& Hunt 2003) a black hole mass of $\log M=6.72 \pm 0.31$, corresponding to $M=5.2 \times 10^{6} M_{\odot}$ with a $68 \%$ confidence interval of $(2.6-11) \times 10^{6} M_{\odot}$. As noted by Burrows et al. (2011), any black hole mass inferred from the $M-L$ relation for Swift $J 164449.3+573451$ is an upper limit because this is the total luminosity of the galaxy, not the bulge. The spectrum of the galaxy indicates that it is star-forming and therefore a spiral galaxy. Since bulge mass is a much better predictor of black hole mass than total luminosity (Kormendy et al. 2011), we can only put an upper limit on the bulge mass and thus on the black hole mass. If the galaxy is bulgeless, it is possible that the black hole mass is much smaller than would be inferred by assuming that the $M-L$ relation.

\section{DISCUSSION}

We have presented a weak but purely observational constraint on the mass of the black hole in the candidate tidal disruption event Swift J164449.3+573451. De-beaming simultaneous radio and X-ray fluxes, and exploiting the ability of refined versions of the fundamental plane of accretion to estimate black hole masses, we find $\log \left(M_{\mathrm{BH}} / M_{\odot}\right)=5.5 \pm 1.1$. This constraint is compatible with the predictions of Rees (1988). The allowed range includes more extreme but exciting interpretations of the event, including the disruption of a white dwarf by a black hole with a mass below $10^{5} M_{\odot}$ (Krolik \& Piran 2011), and it excludes black hole masses that are so large that stars would simply be accreted whole. Tentative evidence of an X-ray quasiperiodic oscillation (QPO) in Swift J164449.3+573451 (Miller $\&$ Strohmayer 2011) implies a mass of $\log \left(M_{\mathrm{BH}} / M_{\odot}\right)=5.7$, assuming the oscillation reflects the Keplerian orbit at the innermost stable circular orbit around a Schwarzschild black hole. It is notable that the central value of the plane-derived mass and the mass implied by the potential QPO are very close.

In this analysis, the source flux was de-beamed using a simple and well-established relationship. This effort benefited from a recent approximation of the Lorentz factor based on radio scintillation (Zauderer et al. 2011). Nevertheless, de-beaming any observed flux is difficult, and prone to uncertainties. The method employed is general in that it can be adapted to any intrinsic source spectral shape; however, difficulties enter when sources are optically thick, with the potential effect of broadening the beaming cone beyond $\theta \simeq 1 / \Gamma$ (e.g., Lind $\&$ Blandford 1985). If this is important in the case of Swift $\mathrm{J} 164449.4+573451$, then the (modest) correction factor may be too high and the mass may be biased to low values. Omitting any beaming correction, our results imply a mass of $\log \left(M_{\mathrm{BH}} / M_{\odot}\right)=6.0 \pm 1.1$; the upper limit is again within the range where massive black holes can disrupt stars.

Additional difficulties enter in that the early X-ray flux of Swift J164449.3+573451 was strongly variable, whereas the radio flux density was comparatively stable (e.g., Levan et al. 2011b; Zauderer et al. 2011). In this and other sources, variability can lead to uncertainties in the mass. Indeed, the nonsimultaneity of many points on the fundamental plane may be an important source of scatter. This analysis attempts to limit related uncertainties by selecting data with at least small periods of strict simultaneity. Moreover, it is worth noting that early spectroscopy by Bloom et al. (2011a) report possible variations in the column density and the possible presence of a blackbody in the spectrum. These may serve to indicate optical depth variations. The column density and simple power-law spectrum measured in the Swift/XRT spectrum used in this analysis are similar to those in later deep observations with XMM-Newton (Miller \& Strohmayer 2011).

A major assumption of our work is that the tidal disruption and accretion of a star by a massive black hole emits radiation as an accretion flow that bears strong similarities to a standard disk, corona, and jet accretion flow geometry that is widely thought to 
hold in AGNs. This is a strong assumption, but it can be tested with future simultaneous X-ray and radio observations. The tentative detection of an X-ray QPO in Swift J164449.3+573451 (Miller \& Strohmayer 2011) may provide some support for this assumption. Because of the rapid variability of this source compared to typical variability timescales for SMBHs, simultaneous $\mathrm{X}$-ray and radio observations in multiple epochs and spanning a decade or more of variation in each parameter would reveal whether or not $L_{R} \sim L_{X}^{0.7}$ (e.g., Gallo et al. 2003). If so, it would strongly suggest that accretion energy from tidal disruption events is radiated in the same way as typical accretion disks and thus validate our approach.

Black hole mass estimates for Swift J164449.3+573451 based on flaring timescales require assumptions about how the timescale associates with orbital timescales, or other timescales in the system, although many of the arguments are compelling (e.g., Krolik \& Piran 2011). Unless the luminosity of host galaxy bulges can be reliably constrained, black hole mass estimates based on the $M-L$ relationship will only give upper limits. The FP mass estimate range of two orders of magnitude is the best observational constraint one can presently give on the black hole in Swift J164449.3+573451, but this could be improved upon with future data and scaling relations.

Currently, the FP is based on only 18 black holes with dynamically measured masses. The rest come from reverberation mapping or secondary scaling relations, such as the $M-\sigma$ relation. The uncertainty in predicting mass is dominated by the intrinsic scatter of the current plane, for which several causes have been identified: (1) X-ray and radio observations from vastly different epochs (Gültekin et al. 2009a; King et al. 2011); (2) heterogeneous observations (observatories, bands, modes, and analysis protocols; Gültekin et al. 2009a); (3) potentially distinct accretion modes (Gültekin et al. 2009a; King et al. 2011; Plotkin et al. 2011); and (4) uncorrected beaming of radiation (Plotkin et al. 2011).

We are now engaged in a joint Chandra and Expanded Very Large Array (EVLA) program to survey all SMBHs with dynamically measured masses. Once complete, the increased numbers $(\sim 60$ sources $)$ will allow us to reduce the effects of the first three sources mentioned above. (The final source of scatter, beaming, has been taken into account for Swift J164449.3+573451.) We estimate that the full sample will reduce the uncertainty in mass to about 0.5 dex, comparable to the $M-\sigma$ and $M-L$ relations (Gültekin et al. 2009b). If a high-quality absorption spectrum can be obtained for the host galaxy of Swift J164449.3+573451, then an $M-\sigma$-based mass is possible. As we noted earlier, without knowing the relative contribution of bulge and disk components, the mass estimate may be too high (Jardell et al. 2011).

More broadly, it is worth noting that masses derived using the fundamental plane may be pragmatic for tidal disruption events. The luminosity of these events means that they can be observed from a broad range of redshifts. At large distances, it will not be possible to obtain direct primary masses, and the combination of distances, timescales, and host galaxy morphology could make it difficult to obtain standard secondary mass estimates. X-ray and radio fluxes are particularly immune to complications such as local obscuration, and often more immune to confusion than optical and NIR observations. New survey efforts, such as PanSTARRS and LSST, will likely detect tidal disruption events in far greater numbers than past efforts. So too will future radio facilities, such as the Square Kilometer Array and LOFAR, and even planned X-ray survey missions such as eROSITA. Given the observational realities and difficulties of tidal disruption events, combining radio and $\mathrm{X}$-ray fluxes to derive black hole masses may prove to be a valuable tool, especially if the derived masses soon equal $M-\sigma$ masses in quality.

K.G. thanks the Aspen Physics Center for their hospitality. J.M.M. and K.G. thank Cole Miller and Doug Richstone for helpful conversations. We thank the anonymous referee for helpful comments that improved this paper.

\section{REFERENCES}

Arnaud, K. A., \& Dorman, B. 2000, XSPEC (available via the HEASARC on-line service at http://heasarc.gsfc.nasa.gov/docs/xanadu/xspec/, provided by NASA/GSFC)

Bloom, J. S., Butler, N. R., Cenko, S. B., \& Perley, D. A. 2011a, GCN Circ., 11847

Bloom, J. S., Giannios, D., Metzger, D., et al. 2011b, Science, 333, 203

Bower, G., Bloom, J., \& Cenko, B. 2011, ATel, 3278

Burrows, D. N., Kennea, J. A., Ghisellini, G., et al. 2011, Nature, submitted (arXiv:1104.4787)

Falcke, H., Kording, E., \& Markoff, S. 2004, A\&A, 414, 895

Gallo, E., Fender, R. P., \& Pooley, G. G. 2003, MNRAS, 344, 60

Gültekin, K., Cackett, E. M., Miller, J. M., et al. 2009a, ApJ, 706, 404

Gültekin, K., Richstone, D. O., Gebhardt, K., et al. 2009b, ApJ, 698, 198

Ho, L. 2008, ARA\&A, 46, 475

Jardell, J., et al. 2011, ApJ, submitted

Jones, S., McHardy, I., Moss, D., et al. 2011, MNRAS, 412, 2641

King, A. L., Miller, J. M., Cackett, E. M., et al. 2011, ApJ, 729, 19

Kormendy, J., Bender, R., \& Cornell, M. E. 2011, Nature, 469, 374

Krolik, J. H., \& Piran, T. 2011, ApJ, submitted (arXiv:1106.0923)

Leloudas, G., Malesani, D., Tanvir, N. R., et al. 2011, GCN Circ., 11830

Levan, A. J., Tanvir, N. R., Wiersma, K., \& Perley, D. 2011 a, GCN Circ., 11833

Levan, A. J., Tanvir, N. R., Cenko, S. B., et al. 2011b, Science, 333, 199

Lind, R., \& Blandford, R. D. 1985, ApJ, 295, L358

Marconi, A., \& Hunt, L. K. 2003, ApJ, 589, L21

Merloni, A., Heinz, S., \& Di Matteo, T. 2003, MNRAS, 345, 1057

Miller, J. M., \& Strohmayer, T. E. 2011, ATel, 3447

Nandra, K., George, I. M., Mushotzky, R. F., Turner, T. J., \& Yaqoob, T. 1997, ApJ, 477, 602

Peacock, J. A. 1999, Cosmological Physics, Cambridge Astrophysics Series (Cambridge: Cambridge Univ. Press), 438

Peterson, B. M., Ferrarese, L., Gilbert, K. M., et al. 2004, ApJ, 613, 682

Plotkin, R. M., Markoff, S., Kelly, B. C., Koerding, E., \& Anderson, S. F. 2011, MNRAS, submitted (arXiv:1105.3211)

Rees, M. J. 1988, Nature, 333, 523

Reines, A. E., Sivakoff, G. R., Johnson, K. E., \& Brogan, C. L. 2011, Nature, 470, 66

Wilms, J., Allen, A., \& McCray, R. 2000, ApJ, 542, 914

Yuan, F., \& Cui, W. 2005, ApJ, 629, 408

Zauderer, B. A., Berger, E., Soderberg, A. M., et al. 2011, Nature, submitted (arXiv:1106.3568) 